\title{
Performance and Energy in Green Superposition Coding Wireless Networks: An Analytical Model
}

\author{
Jean-Marc Kelif ${ }^{1}$, Jean-Marie Gorce ${ }^{2}$, Azeddine Gati ${ }^{3}$
}

\begin{abstract}
In this paper, we develop a powerful analytical model of wireless network with Superposition Coding (SC), also referred to as Non Orthogonal Multiple Access (NOMA). This model allows to establish a closed form expression of the minimum power a base station (BS) need to transmit, for a user to achieve a given SINR (signal to interference plus noise ratio) whatever its location in the area covered by the base station. It moreover allows to establish a closed form expression of the minimum total transmit power of a base station. These closed form expressions allow to establish performance of wireless networks, by minimizing the base stations transmit powers. As an application, we show that these closed form expressions allow to quantify the energetic performance, spectral efficiency, total throughput and the coverage of a BS, in a simple and quick way.
\end{abstract}

\section{INTRODUCTION}

The design of future $5 \mathrm{G}$ wireless networks has to address new challenges such as a latency reduction and capacity increase. In addition, the possibility to connect billions objects receiving data, related to the Internet of Things (IoT), represents also an important issue of the 5G.

In order to meet these requirements, different solutions are envisioned by telecommunication operators allowing to increase networks capacity. Among them, the Superposition Coding (SC) mechanism may represent an interesting approach. Knowing that the achievable throughput of wireless systems is bounded by a fundamental limit [1] [2] [4], one interest of SC, also referred to as Non Orthogonal Multiple Access (NOMA) [3], is that it achieves this fundamental bound, providing more capacity than classical orthogonal multiple access [4] [5].

$\mathrm{SC}$ is also promising in the case of IoT, since data need to be transmitted, simultaneously, towards a great number of communicating objects. Several network access technologies have recently been proposed for IoT, as analyzed in [6] [7], and in uplink scenario [8] [9]. An important open question of IoT is to determine the maximum user density that a base station (BS) can serve, taking into account the transmit power, performance and coverage constraints. Considering a spatial density of users, a new approach has been proposed in [4] [5], called spatial continuum model. Based on SC, this approach allowed to analyze the fundamental limits of a dense cell.

\footnotetext{
${ }^{1}$ Jean-Marc Kelif is with Orange Labs, France. E-mail: jeanmarc.kelif@orange.com

${ }^{2}$ Jean-Marie Gorce is with Univ Lyon, INSA Lyon, Inria, CITI, Villeurbanne, France. E-mail: jean-marie.gorce@insa-lyon.fr. J-M Gorce is partly supported by a CRE with Orange.

${ }^{3}$ Azeddine Gati is with Orange Labs, France. E-mail: azeddine.gati@orange.com
}

In [13] $\mathrm{SC}$ is shown to be capacity achieving for a continuous-time Poisson broadcast channel with different parameters.

Analyzing a scenario with two users, one close to a BS and the other far from it, the paper [14] considers multicast beamforming with superposition coding. An iterative algorithm is developed, based on an optimal power allocation, in order to find beamforming vectors and powers for both users.

In the opposite, assuming Gaussian broadcast channels and taking into account the receiver power constraints, [15] shows that multi-user transmission with SC is not always optimal in terms of spectral efficiency.

In [16] SC is used in the aim to increase the system capacity through multiuser diversity exploitation. A joint admission control and SC scheme is proposed which allows to provide a good tradeoff between the QoS level perceived by the user in the system and the utilization of the scarce radio resources.

The paper [17] develops an analysis of NOMA scheme in the uplink of wireless powered communication networks. The objective is to maximize the sum rate by jointly designing the time allocation, the downlink energy beamforming and receiver beamforming. It moreover develops an algorithm in the aim to obtain the optimal time allocation. Authors establish results which outperform the fixed time allocation.

Considering the downlink of a NOMA wireless network, authors of [18] develop resource allocation algorithms Their algorithms are energy-efficient. In their approach, they assign only two users to the same subchannel. Their analysis allows to maximize the system energy efficiency.

None of these papers proposes closed form formulas of the BS transmit power towards a user and total BS transmit power.

Our Contribution: We develop an analytical model allowing to analyze a wireless network with superposition coding, in which successive interference cancellation (SIC) is implemented. Our analysis takes into account the interference induced by all the base stations of the network. We establish a closed form formula of the minimum power a BS needs to transmit, for a user to reach a SINR target, whatever its location in the area covered by the base station. Moreover, we establish a closed form formula of the minimum BS total transmit power. To the best of our knowledge, this is the first time such formulas are established. This allows to analyze in a simple and quick way, the performance of base stations in terms of energy and throughput, and their coverage. 
The organization of the paper follows. In Section II the system model is presented. In Section III] we establish the closed form formulas of the BS transmit power towards a user whatever its location, and the total base station transmit power. Section IV proposes an analysis of these closed form formulas. Section $\mathrm{V}$ presents an application of these closed form formulas focused on the analysis of coverage and performance, in terms of energy and throughput. Section VI concludes the paper.

\section{System MODEL}

We consider a wireless network made of $M$ base stations uniformly distributed. All base stations transmit over the same frequency bandwidth. We focus our analysis on the downlink, on the area covered by a BS $b$, using a superposition coding mechanism. Moreover the successive interference cancellation (SIC) is implemented. Let remind that superposition coding is also referred to as Non Orthogonal Multiple Access (NOMA) [3], Let us consider:

- $\mathcal{M}=\{1, \ldots, M\}$ the set of base stations, uniformly and regularly distributed over the two-dimensional plane.

- $U=\{1, \ldots, U\}$ the set of users uniformly distributed over the network.

- $N=\{1, \ldots, N\}$ the set of users connected to a BS, uniformly and regularly distributed.

- $\gamma^{*}$ (also denoted $\gamma$ ) the SINR target to be reached in downlink by any receiver (user).

- $r_{u}$ the distance between the transmit BS $b$ and the user $u$, connected to $b$.

- $N_{u}$ the set of users connected to $b$, located at a distance lower or equal to the distance $r_{u}$.

- $\bar{N}_{u}$ the set of users connected to $b$, located at a distance greater than $r_{u}$.

- $\quad P_{u}$ the transmitted power assigned by the base station $b$ towards user $u$.

- $g_{u}$ the propagation gain between the serving BS $b$ and user $u$.

Let consider that a SINR target, denoted $\gamma^{*}$, has to be reached at each user, in the aim to reach a given throughput. We aim to establish a mathematical expression of a BS transmit power, in the aim to reach this SINR target. Since users and base stations are uniformly distributed over the network, we can focus the analysis on any given BS.

\section{A. Expression of the SINR with Superposition Coding}

In our analysis, by considering $\mathrm{SC}$, we assume that a $\mathrm{BS}$ transmits useful power to all the users of the cell it covers, simultaneously, on the same frequency bandwidth.

Considering a given BS denoted $b$, we can express the total amount of power received by any user (UE) $u$ connected to the BS $b$, as the sum of: (i) a useful signal $P_{u} g_{u}$, (ii) the interference power due to the transmit powers of the BS $b$ towards the other users of the cell: $\sum_{v \in N, v \neq u} P_{v} g_{u}$, where $P_{u}$ and $P_{v}$ are the transmit powers towards the mobile $u$ and $v$ and $g_{u}$ is the pathloss between the BS $b$ and the mobile $u$, (iii) the interference power due to the total transmit power of the other BSs $j \neq b$ of the network which use the same frequency banwidth as $b: I_{N w}=\sum_{j \neq b} P_{j} g_{j, u}$ where $P_{j}$ is the total transmit power of BS $j$ towards their own users and $g_{j, u}$ is the propagation gain between BS $j$ and the mobile $u$ (connected to $b$ ), (iv) and thermal noise power $N_{t h}$. Therefore, we can consider the SINR target $\gamma^{*}(u)$ defined by:

$$
\gamma^{*}(u)=\frac{P_{u} g_{u}}{\sum_{v \in N, v \neq u} P_{v} g_{u}+I_{N w}+N_{t h}} .
$$

Users are connected to their best server BS, i.e. the BS which provides the best useful signal. Therefore, the shadowing can be neglected as shown in [11] [12]. Let remind that, in this case, the best server BS is the nearest BS.

Therefore, considering $g_{v}=K r_{v}^{-\eta}$ [10], the SINR is expressed as:

$$
\gamma^{*}(u)=\frac{P_{u} K r_{u}^{-\eta}}{\sum_{v \in N, v \neq u} P_{v} K r_{u}^{-\eta}+I_{N w}+N_{t h}},
$$

where $\eta$ is the pathloss parameter and $\mathrm{K}$ is a constant.

\section{B. Successive Interference Cancellation}

Since the BS transmits useful powers simultaneously to all the users, and on the same frequency, a user $u$ receives a high level of interference due to the powers transmitted towards all the other users of the cell. The SIC mechanism allows to mitigate this interference. Let rewrite the expression (2) by considering the set of users $N$ as the union of the set $N_{u}$ of users $v$, such that $r_{v} \leq r_{u}$ and the set $\bar{N}_{u}$ of users $v$, such that $r_{v}>r_{u}$ :

$$
\gamma^{*}(u)=\frac{P_{u} K r_{u}^{-\eta}}{\sum_{v \in N_{u}, v \neq u} P_{v} K r_{u}^{-\eta}+\sum_{v \in \bar{N}_{u}, v \neq u} P_{v} K r_{u}^{-\eta}+I_{N w}+N_{t h}}
$$

For the user $u$ to reach a given target $\operatorname{SINR} \gamma^{*}$, it is needed to transmit at a given power $P_{u}$. Due to the pathloss between a transmitter and a receiver, users located at distances $r_{v}>r_{u}$ (from BS b) need a transmit power $P_{v}>P_{u}$ in the aim to reach the same $\operatorname{SINR} \gamma^{*}$. The successive interference cancellation mechanism allows the user $u$ to decode, using an iterative process, the powers it receives dedicated to users located at distances further than him (from the BS b). Let notice $p_{u}^{u}$ the power dedicated to user $u$ and received by the user $u\left(p_{u}^{u}=P_{u} K r_{u}^{-\eta}\right)$. Let notice $p_{u}^{v}$ the power dedicated to user $v$ and received by the user $u\left(p_{u}^{v}=P_{v} K r_{u}^{-\eta}\right)$.

Let consider a user $u$ needing a useful power $p_{u}^{u}$ in the aim to reach the SINR $\gamma^{*}$. Using the SIC mechanism, the user $u$ is able to decode any (interfering) power it receives higher than $p_{u}^{u}$. And particularly, it is able to decode the power it receives dedicated to the user $v$. Indeed, since $P_{v} \geq P_{u}$, the power $p_{u}^{v}$ has a higher value than $p_{u}^{u}$. Therefore, $p_{u}^{v}$ can be decoded by the user $u$. And thus, it does no more represent an interference for $u$. This is available for any user $v$ for which 


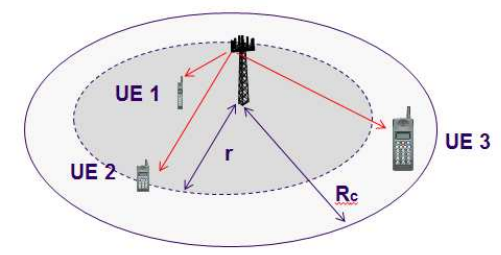

Figure 1. Cell of radius $R_{c}$. SIC: user UE 2, located at r, is able to decode the power dedicated to any user UE 3, located at a distance greater than $r$ (white area). The power dedicated to any user UE 1, located at a distance lower than $\mathrm{r}$ (grey area), cannot be decoded by user UE 2 .

$r_{v}>r_{u}$. As a consequence, considering all the users of the cell, , the term $\sum_{v \in \bar{N}_{u}, v \neq u} P_{v} K r_{u}^{-\eta}$ disappears from expression (3), since it does no more represent an interference (see fig. 11). We thus can write (3) as:

$$
\gamma^{*}(u)=\frac{K P_{u} r_{u}^{-\eta}}{\sum_{v \in N_{u}, v \neq u} K P_{v} r_{u}^{-\eta}+I_{N w}+N_{t h}} .
$$

which can be written:

$$
\gamma^{*}(u)=\frac{P_{u}}{\sum_{v, r_{v} \leq r_{u}} P_{v}-P_{u}+\frac{I_{N w}+N_{t h}}{K} r_{u}^{\eta}} .
$$

From this expression, the total power transmitted to users located at a distance lower or equal to $r_{u}$ can be expressed as:

$$
\sum_{v, r_{v} \leq r_{u}} P_{v}=\frac{\gamma^{*}(u)+1}{\gamma^{*}(u)} P_{u}-\frac{I_{N w}}{K} r_{u}^{\eta}-\frac{N_{t h}}{K} r_{u}^{\eta}
$$

Since $I_{N w}=\sum_{j \neq b} P_{j} g_{j, u}=\sum_{j \neq b} K P_{j} r_{j, u}^{-\eta}$, we can write

$$
\sum_{v, r_{v} \leq r_{u}} P_{v}=\frac{\gamma^{*}(u)+1}{\gamma^{*}(u)} P_{u}-\sum_{j \neq b} P_{j} r_{j, u}^{-\eta} r_{u}^{\eta}-\frac{N_{t h}}{K} r_{u}^{\eta}
$$

From this expression, we can express the transmit power towards a user and the total transmit power of a BS.

\section{Wireless Network ANAlytical Model}

The analytical model proposed in this paper consists in considering the interfering base stations of the network as a continuum of base stations, characterized by a density of base station $\rho_{b s}$. This approach was validated by comparison with a simulated hexagonal network [19] [20] [21]. Considering a uniform distribution of users over the network, we can write $\mathrm{P}_{j}=\mathrm{P}_{B}=$ constant. Therefore, the interference due to all the base stations of the network $\sum_{j, j \neq b} K P_{B} r_{j, u}^{-\eta}$ can be expressed as $\int P_{B} \rho_{b s} K s^{-\eta} s d s d \theta$, where $s d s d \theta$ represents the element of integration. The approach developed in [19] [20] [21] allows to express this integral as $2 \pi \frac{\rho_{b s} P_{B} K\left(2 R_{c}-r_{u}\right)^{2-\eta}}{\eta-2}$, where $2 R_{c}$ represents the intersite distance (ISD) between two BS. We refer the reader to [19] [20] [21] for the detailed explanation and validation through Monte Carlo simulations. Using this model, it becomes possible to express (7) as follows. Assuming the cell covered by the $\mathrm{BS}$ as a disk of radius $R_{c}$, we consider a user located at a distance $r$ from it (dropping the index $u$ ). The discrete sum on users $v$ of (7) can be expressed as an integral over the cell. A unique SINR target $\gamma^{*}$ is considered for all the users of the cell. This corresponds to a service which demands a given throughput for all users. For sake of simplicity, let denote $\gamma=\gamma^{*}$ and $\zeta=\frac{\gamma^{*}+1}{\gamma^{*}}$. We can write (7) as:

$$
\begin{aligned}
\int_{0}^{2 \pi} \int_{R_{0}}^{r} \rho(s) P(s) s d s d \theta= & \zeta P(r) \\
& -\frac{2 \pi \rho_{b s}\left(2 R_{c}-r\right)^{2-\eta} r^{\eta}}{\eta-2} P_{B} \\
& -\frac{N_{t h}}{K} r^{\eta}
\end{aligned}
$$

where $P(r)$ is the power transmitted towards the user located at distance $r, s$ is the variable of integration, $\rho(s)$ is the density of users on the area covered by the BS, depending on $\mathrm{s}$ and $s d s d \theta$ is the element of integration, and $R_{0}$ the minimum distance between a user and the base station.

Let remind that in expression (8) the parameter $P_{B}$, which represents the total transmit power of a BS, is a constant. Since the users are uniformly distributed over the area of the cell, $\rho(s)=\rho$ does not depend on $s$, (8) can be written as:

$$
\begin{aligned}
2 \pi \rho \int_{R_{0}}^{r} P(s) s d s= & \zeta P(r)-\frac{2 \pi \rho_{b s}\left(2 R_{c}-r\right)^{2-\eta}}{\eta-2} r^{\eta} P_{B} \\
& -\frac{N_{t h}}{K} r^{\eta} .
\end{aligned}
$$

Let denote

$$
\begin{gathered}
a(r)=2 \pi \frac{\rho_{b s}\left(2 R_{c}-r\right)^{2-\eta}}{\eta-2} r^{\eta}, \\
b(r)=\frac{N_{t h}}{K} r^{\eta},
\end{gathered}
$$

and

$$
F(r)=a(r) P_{B}+b(r) .
$$

We thus can write (9) as:

$$
2 \pi \rho \int_{R_{0}}^{r} P(s) s d s=\zeta P(r)-F(r) .
$$

From expression (13), two important expressions can be derived:

i) the transmit power towards a user located at distance $r$,

ii) the total BS transmit power.

The two following theorems can be written:

\section{A. Theorem 1: Transmit Power Towards A User}

Considering a density of users $\rho$, and assuming a unique SINR target $\gamma$ over the cell, the transmit power towards a user located at a distance $r$ from the BS, denoted $P(r)$, can be expressed as (denote $\alpha=\frac{\gamma}{\gamma+1} \rho \pi$ ):

$$
P(r)=\frac{\gamma}{\gamma+1} e^{\alpha r^{2}} \int_{R_{0}}^{r} F^{\prime}(s) e^{-\alpha s^{2}} d s
$$


where $F^{\prime}(r)$ is the derivative of $F(r)$ with respect to $r$ :

$$
F^{\prime}(r)=a^{\prime}(r) P_{B}+b^{\prime}(r)
$$

and $a^{\prime}(r)=4 \pi \frac{\rho_{b s}\left(R_{c} \eta-r\right)\left(2 R_{c}-r\right)^{1-\eta} r^{\eta-1}}{\eta-2}$ is the derivative of $\mathrm{a}(\mathrm{r})$ with respect to $\mathrm{r}$, and $b^{\prime}(r)=\eta \frac{N_{t h}}{K} r^{\eta-1}$ is the derivative of $b(r)$ with respect to $r$.

The proof is detailed in Appendix 1.

\section{B. Theorem 2: Total BS Transmit Power}

Considering a density of users $\rho$, and assuming a unique SINR target $\gamma$ over the cell, the total power transmitted by the BS, simultaneously towards all the users of the cell, denoted $P_{B}$, can be expressed as:

$$
P_{B}=\frac{e^{\alpha R_{c}^{2}} \int_{R_{0}}^{R_{c}} b^{\prime}(s) e^{-\alpha s^{2}} d s-b\left(R_{c}\right)}{1-e^{\alpha R_{c}^{2}} \int_{R_{0}}^{R_{c}} a^{\prime}(s) e^{-\alpha s^{2}} d s+a\left(R_{c}\right)},
$$

The proof is detailed in Appendix 2.

\section{Remark}

Let notice that if the interference due to the other base stations of the network is neglected, the term $a(r)$ is neglected. The total BS transmit power can thus be expressed as:

$$
P_{B}=e^{\alpha R_{c}^{2}} \int_{R_{0}}^{R_{c}} b^{\prime}(s) e^{-\alpha s^{2}} d s-b\left(R_{c}\right) .
$$

Since $b^{\prime}(s)=\eta \frac{N_{t h}}{K} s^{\eta-1}$ (from (11)), we can write

$$
P_{B}=\eta \frac{N_{t h}}{K} e^{\alpha R_{c}^{2}} \int_{R_{0}}^{R_{c}} s^{\eta-1} e^{-\alpha s^{2}} d s-\frac{N_{t h}}{K} R_{c}^{\eta}
$$

By considering $R_{0}<<R_{c}$ and thus $\frac{R_{0}}{R_{c}} \approx 0$, and denoting $\beta=\alpha R_{c}^{2}$, we can express (18) as:

$$
P_{B}=2 \beta \frac{N_{t h}}{K} R_{c}^{\eta} e^{\beta}\left(\Gamma\left(\frac{\eta+2}{2}, \beta\right)-\Gamma\left(\frac{\eta+2}{2}\right)\right)
$$

where $\Gamma(x)=\int_{0}^{\infty} t^{x-1} e^{-t} d t$ and $\Gamma(u, x)=\int_{x}^{\infty} t^{u-1} e^{-t} d t$ is the incomplete $\Gamma$ function.

\section{Base Station Transmit Power Analysis}

\section{A. Transmit Power Expressions Interest}

The expressions (14) and (16) highlight the impact of the parameters of the whole network

- on the transmit power towards a user located at a distance $r$ (14),

- on the total transmit power of the BS (16).

The minimum transmitted power needed to reach a given SINR target $\gamma^{*}$ depends on

i) the user location $r$ (transmit power towards a user (14)),

ii) the size of the cell $R_{c}$ (total transmit power (16)),

iii) the propagation (pathloss factor) $\eta$,

iv) the antenna characteristics and frequency (parameter $K$ ), v) the thermal noise $N_{t h}$.

vi) the density of users and the SINR target value.

Considering the network impact, two more parameters play a role: the intersite distance and the density of BS.

\section{B. Network Impact}

It can be noticed that the network impact is expressed as the denominator of (16). Since a power is a positive quantity, the denominator of needs to be strictly positive:

$$
1-e^{\alpha R_{c}^{2}} \int_{R_{0}}^{R_{c}} a^{\prime}(s) e^{-\alpha s^{2}} d s+a\left(R_{c}\right)>0 .
$$

If the denominator is close to 0 , the power tends to infinity. From (10), since $\rho_{b s}=\frac{1}{\pi R_{c}^{2}}$, we can express (20) as:

$$
\frac{4}{\eta} e^{\beta} \int_{R_{0} / R_{c}}^{1}(\eta-u)(2-u)^{1-\eta} u^{\eta-1} e^{-\beta u^{2}} d u<1
$$

This expression shows constraints on the density of users and the SINR target. Moreover, they are strongly correlated (since $\beta=\alpha R_{c}^{2}=\frac{\gamma}{\gamma+1} \rho \pi R_{c}^{2}$ ). The SINR target is limited for a given density of users. Therefore the achievable throughput is limited. This means that the behavior observed (fig.2 and fig. 3, dotted curves) is directly induced by the network impact. Over a given SINR target value, the needed transmit power increases indefinitely. For example, fig. 2 (left side, dotted curve) shows that for a density of 8 users per cell, and a cell radius of $50 \mathrm{~m}$, the BS transmit power varies from -15 to $29 \mathrm{dBm}$ for a total cell throughput varying from $1 \mathrm{Mbits} / \mathrm{s}$ to a maximum of $30 \mathrm{Mbits} / \mathrm{s}$. Let remind that this value corresponds to a given value of SINR. And then, the transmit power value increases indefinitely, without increasing the throughput.

\section{Isolated Cell}

Considering a theoretic case where the impact of the network could be neglected, the SINR target and the density of users could reach high level values. Expression (18) shows that the density of users and the SINR target are not limited.

The examples of fig. 2 and 3 show that the curves without network impact (plain curves) have behaviors completely different than the ones with the impact of network (dotted curves). Without network impact, the reachable SINR target is not limited. It can reach very high values. Therefore, the total throughput can reach, in theory, very high values, by using limited transmit powers. Therefore, the network impact is significant. Let notice that the isolated cell represents a theoretic case, which does not exist (or whose probability is very low) in a real network.

These powerful expressions make it possible to develop network analyzes of great interest in a simple and quick analytical way. Hereafter, we propose an example of application focused on performance and energy. Other applications may be developed such as, for example, dimensioning or outage probability analysis. 

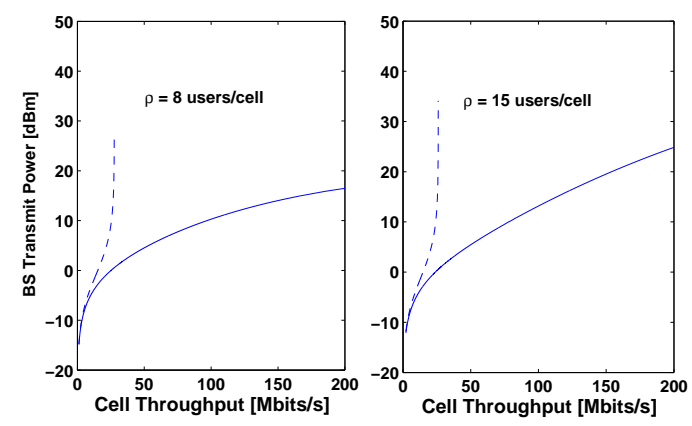

Figure 2. Base station Transmit Power vs Total throughput of the cell. Comparison for a density of 8 users per cell area (left) and 15 users per cell area (right), and for a radius of the zone covered of $R_{c}=50 \mathrm{~m}$, considering the impact of the network (dotted curve) and neglecting the impact of the network (plain curve).
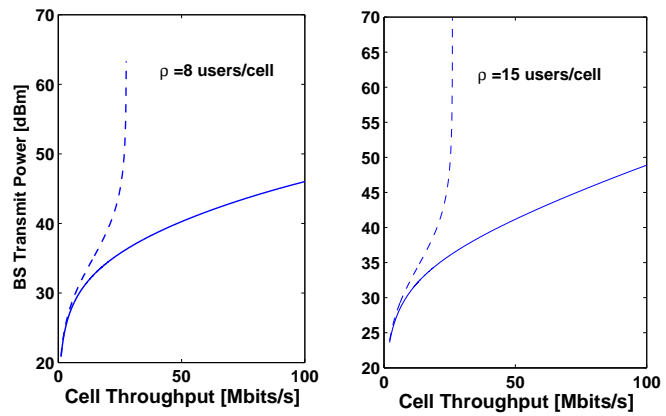

Figure 3. Base station Transmit Power vs Total throughput of the cell. Comparison for a density of 8 users per cell area (left) and 15 users per cell area (right), and for a radius of the zone covered of $R_{c}=500 \mathrm{~m}$, considering the impact of the network (dotted curve) and neglecting the impact of the network (plain curve).

\section{Application: Energy, Performance And COVERAGE}

In this section, the energetic performance, throughput, and coverage of a BS, are analyzed and quantified in an analytical way (considering values given by Table @). The minimum transmit power needed for a BS to cover an area of radius $R_{c}$ is given by expression (16). In other words the coverage can directly be calculated with accuracy by using (16), for a given transmit power. And moreover, this allows to minimize the energy needed to cover a given area. This expression also gives the reachable level of SINR in the cell, and consequently the maximum throughput. Let remind that, for a given SINR $\gamma$, the spectral efficiency SE is given by $\mathrm{SE}=\log _{2}(1+\gamma)$ and the throughput $\mathrm{D}$ is given by $\mathrm{D}=W \log _{2}(1+\gamma)$, where $\mathrm{W}$ is the frequency bandwidth.

Fig. 2 and 3 compare the reachable performances, in terms of energy and total throughput per cell, by considering or not, the interference induced by the network.

1) Considering the network impact : the maximum total throughput of the cell reaches $25 \mathrm{Mbits} / \mathrm{s}$ (Fig. 2 and 3, dotted curves), for 8 and 15 users/cell area, for $R_{c}=50 \mathrm{~m}$ (Fig. 2)
Table I

VALUE PARAMETERS

\begin{tabular}{|c|c|}
\hline Cell radius $R_{c}$ & $50 \mathrm{~m}-500 \mathrm{~m}$ \\
\hline User distribution & Uniform \\
\hline Densities of users/cell area $\rho$ & $8-15$ \\
\hline Frequency bandwidth $\mathrm{W}$ & $10 \mathrm{MHz}$ \\
\hline Propagation parameters & $K=2.68 \times 10^{-4} ; \eta=3.57$ \\
\hline Noise density power $N_{t h}$ & $-166 \mathrm{dBm} / \mathrm{Hz}$ \\
\hline
\end{tabular}

and $R_{c}=500 \mathrm{~m}$ (Fig. 3). The BS transmit power needed to reach the maximum throughput of $25 \mathrm{Mbits} / \mathrm{s}$ increases with $R_{c}$. It reaches about $10 \mathrm{dBm}$ for $R_{c}=50 \mathrm{~m}$, and $46 \mathrm{dBm}$ for $R_{c}=500 \mathrm{~m}$. Therefore, the expression (16) also allows to show that the transmit power is an increasing function of the total throughput. Moreover, it increases with $R_{c}$, too.

2) Without network impact : the total throughput of the cell can theoretically increase indefinitely (Fig. 2 and 3 , plain curves). In the aim to quantify the network impact, we consider the same transmitting values as before, $10 \mathrm{dBm}$ and $46 \mathrm{dBm}$. For $R_{c}=50 \mathrm{~m}$ (Fig. 2), and a BS transmit power of $10 \mathrm{dBm}$, a total throughput of $100 \mathrm{Mbits} / \mathrm{s}$ is reached for a density of 8 users per cell area (resp. $80 \mathrm{Mbits} / \mathrm{s}$ for 15 users per cell area). For $R_{c}=500 \mathrm{~m}$ (Fig. 3), and a BS transmit power of $46 \mathrm{dBm}$, a total throughput of $100 \mathrm{Mbits} / \mathrm{s}$ is reached for a density of 8 users per cell area (resp. 80 Mbits/s for 15 users per cell area). Therefore, due to network impact, a decrease of up to $75 \%$ of the total throughput is observed in Figures 2 and 3. This confirms the significant impact of the network.

\section{CONCLUSION}

We establish closed form expressions of the minimum total transmit power of a BS and of the transmit power needed to achieve a given SINR, whatever the user location, in a wireless network, by considering the Superposition Coding and SIC mechanism. These expressions allow to highlight the impact of the parameters of the system such as the cell radius, the propagation parameters, and the intersite distance. In particular they allow taking into account the impact of the whole network. These expressions are powerful since they make it possible to carry out analyzes in a simple and quick way. As an application, we quantified with accuracy the energetic performance, throughput and coverage of a base station. In a future work, the impact of a dynamic traffic scenario will be analyzed.

\section{APPENDIX 1}

First, the method to determine the transmitting power consists in derivating expression (13) with respect to $r$. It thus can be written:

$$
2 \pi \rho P(r) r=\zeta P^{\prime}(r)-F^{\prime}(r),
$$

where $P^{\prime}$ (resp. $F^{\prime}$ ) means the derivative of $P$ (resp. $F$ ) with respect to $r$. This differential equation with non-constant coefficients allows to express the transmit power towards a user located at a distance $r$ from its serving BS as:

$$
P(r)=C(r) e^{\frac{\gamma}{\gamma+1} \rho \pi r^{2}}
$$


where

$$
C(r)=\frac{\gamma}{\gamma+1} \int_{R_{0}}^{r} F^{\prime}(s) e^{-\frac{\gamma}{\gamma+1} \rho \pi s^{2}} d s
$$

\section{APPENDIX 2}

The total transmit power of a BS is expressed as:

$$
\begin{aligned}
P_{B} & =\int_{0}^{2 \pi} \int_{R_{0}}^{R_{c}} \rho P(s) s d s d \theta \\
& =2 \pi \rho \int_{R_{0}}^{R_{c}} P(s) s d s \\
& \stackrel{(3)}{=} \zeta P\left(R_{c}\right)-F\left(R_{c}\right) .
\end{aligned}
$$

In expression (25), the third equality denoted (3) comes from (13), where $\mathrm{r}=\mathrm{R}_{c}$. We denote $\alpha=\frac{\gamma}{\gamma+1} \rho \pi$. Let express $\zeta P\left(R_{c}\right)$ from 23):

$$
\zeta P\left(R_{c}\right)=\zeta C(R c) e^{\alpha R_{c}^{2}}
$$

where (from 24), we can write (remind that $\zeta=\frac{\gamma+1}{\gamma}$ ):

$$
\zeta C\left(R_{c}\right)=\int_{R_{0}}^{R_{c}} F^{\prime}(s) e^{-\alpha s^{2}} d s
$$

From (12), we can express:

$$
F(r)=a(r) P_{B}+b(r),
$$

where $a(r)=2 \pi \frac{\rho_{b s}\left(2 R_{c}-r\right)^{2-\eta}}{\eta-2} r^{\eta}$ and $b(r)=\frac{N_{t h}}{K} r^{\eta}$.

Therefore $F^{\prime}(r)$ can be expressed as:

$$
F^{\prime}(r)=a^{\prime}(r) P_{B}+b^{\prime}(r)
$$

And thus, we can express:

$$
\zeta C\left(R_{c}\right)=A\left(R_{c}\right) P_{B}+B\left(R_{c}\right)
$$

where

$$
A\left(R_{c}\right)=\int_{R_{0}}^{R_{c}} a^{\prime}(s) e^{-\alpha s^{2}} d s
$$

and

$$
B\left(R_{c}\right)=\int_{R_{0}}^{R_{c}} b^{\prime}(s) e^{-\alpha s^{2}} d s
$$

From expressions 25] to 32, we can express the total BS transmit power as:

$$
\begin{aligned}
P_{B}= & \zeta P\left(R_{c}\right)-F\left(R_{c}\right) \\
= & \zeta C\left(R_{c}\right) e^{\alpha R_{c}^{2}}-F\left(R_{c}\right) \\
= & A\left(R_{c}\right) e^{\alpha R_{c}^{2}} P_{B}+B\left(R_{c}\right) e^{\alpha R_{c}^{2}} \\
& -a\left(R_{c}\right) P_{B}-b\left(R_{c}\right)
\end{aligned}
$$

And thus:

$$
P_{B}=\frac{B\left(R_{c}\right) e^{\alpha R_{c}^{2}}-b\left(R_{c}\right)}{1-A\left(R_{c}\right) e^{\alpha R_{c}^{2}}+a\left(R_{c}\right)} .
$$

From (31) and 32, we can write

$$
P_{B}=\frac{e^{\alpha R_{c}^{2}} \int_{R_{0}}^{R_{c}} b^{\prime}(s) e^{-\alpha s^{2}} d s-b\left(R_{c}\right)}{1-e^{\alpha R_{c}^{2}} \int_{R_{0}}^{R_{c}} a^{\prime}(s) e^{-\alpha s^{2}} d s+a\left(R_{c}\right)},
$$

\section{REFERENCES}

[1] C. Shannon, A mathematical theory of communication, Bell Sys. Tech. J., vol. 27, pp. 379423, 1948.

[2] A. D. Wyner, Shannon-theoretic approach to a gaussian cellular multipleaccess channel, IEEE Transactions on Information Theory, vol. 40, no. 6, pp. 17131727, 1994.

[3] Z. Ding, Z. Yang, P. Fan, and H. V. Poor, On the performance of non-orthogonal multiple access in $5 \mathrm{~g}$ systems with randomly deployed mobiles, IEEE Signal Processing Letters, vol. 21, no. 12, pp. 15011505 , 2014.

[4] J.-M. Gorce, J.-M. Kelif, and H. V. Poor, Spatial continuum model: Toward the fundamental limits of dense wireless networks, in Proc. IEEE Globecom), 2016.

[5] J.-M. Gorce, D. Tsilimantos, P. Ferrand, and H. V. Poor, Energy-capacity trade-off bounds in a downlink typical cell, in Proc. IEEE Int. Symp. on Personal, Indoor and Mobile Radio Commun.(PIMRC), 2014.

[6] C. Goursaud and J.-M. Gorce, Dedicated networks for iot: Phy/mac state of the art and challenges, EAI endorsed transactions on Internet of Things.

[7] M. Centenaro, L. Vangelista, A. Zanella, and M. Zorzi, Long-range communications in unlicensed bands: The rising stars in the IoT and smart city scenarios, IEEE Trans. Wireless Com., 2016.

[8] V. Chandrasekhar and J. G. Andrews, Uplink capacity and interference avoidance for two-tier femtocell networks, IEEE Transactions on Wireless Communications, vol. 8, no. 7, pp. 34983509, 2009.

[9] H. S. Dhillon, H. Huang, H. Viswanathan, and R. A. Valenzuela, Fundamentals of throughput maximization with random arrivals for M2M communications, Communications, IEEE Transactions on, vol. 62, no. 11, pp. 40944109, 2014.

[10] Report ITU-R M.2135-1, Guidelines for evaluation of radio interface technologies for IMT-Advanced, 12/2009

[11] J-M Kelif, M. Coupechoux,, F. Marache, Limiting the Power of 4G Dynamic Green Cellular Networks: Impact on Capacity and Quality of Service, Sarnoff Symposium- September 2015, Newark, USA

[12] Using Poisson processes to model lattice cellular networks B. Baszczyszyn, M K. Karray and H. P. Keeler Arxiv:1207.7208v2, 2013

[13] H. Kim, B. Nachman, A. El Gamal 'Superposition Coding is Almost Always Optimal for the Poisson Broadcast Channel', ISIT International Conference, 2015

[14] J. Choi, Minimum Power Multicast Beamforming With Superposition Coding for Multiresolution Broadcast and Application to NOMA Systems IEEE TRANSACTIONS ON COMMUNICATIONS, VOL. 63, NO. 3, MARCH 2015

[15] M. Kim and H. Viswanathan, Energy-Efficient Delay-Tolerant Communication: Revisiting Optimality of Superposition Coding in Broadcast Channels, Globecom 2014

[16] C-H Hwang, M. Wu, T-P Low, Y-J Liao, and L-S Tsai, Performance Evaluation of Superposition Coding in Downlink LTE, VTC Fall, 2015

[17] Y. Abbes, S. Najeh and H. Besbes, Joint Superposition Coding and Great Fairness Admission Control for QoS provisioning in LTE systems, IWCMC, 2016

[18] F. Fang, H. Zhang, J. Cheng, V. C. M. Leung, 'Energy-Efficient Resource Allocation for Downlink Non-Orthogonal Multiple Access Network' IEEE TRANSACTIONS ON COMMUNICATIONS, VOL. 64, NO. 9 , SEPTEMBER 2016

[19] J-M. Kelif and E. Altman, Downlink Fluid Model of CDMA Networks, Proc. of IEEE VTC Spring, May 2005.

[20] Jean-Marc Kelif, Marceau Coupechoux, Philippe Godlewski, A Fluid Model for Performance Analysis in Cellular Networks, EURASIP Journal on Wireless Communications and Networking, Vol. 2010, Article ID 435189, doi:10.1155/2010/435189

[21] J.-M. Kelif, M. Coupechoux and P. Godlewski, Spatial Outage Probability for Cellular Networks, Proc. of GLOBECOM, 2007. 